\title{
Introduction to the special issue for SIMAI 2016
}

\author{
Valeria Ruggiero ${ }^{1} \cdot$ Gerardo Toraldo $^{2}$ \\ Published online: 27 June 2018 \\ (c) Springer Science+Business Media, LLC, part of Springer Nature 2018
}

In 2016 the biennial congress of the Italian Society of Industrial and Applied Mathematics (SIMAI) was held at Politecnico di Milano (Italy). Promoting collaboration between mathematicians, industrial practitioners and management scientists is among the main goals of SIMAI. In Milan, a number of minisymposia were dedicated to applications and computational aspects of numerical optimization. They were motivated by the historically established interplay between numerical optimization and application problems [3,9], aimed at improving the design, planning and/or performances of processes in industry or management. More recently, optimization has assumed great importance for the extraction of information from very large data sets and, in particular, for signal, image and learning processing in a myriad of application areas.

In the optimization problems arising in many real life problems, the design of effective algorithms is a challenging task, either because of the high computational cost of evaluating objectives and constraints, or because of the nonlinearity, multimodality, discontinuity and uncertainty in the data and in the models. Thus the effective solution of practically significant problems is based on a blend of theoretical and computational issues.

The contributions from the SIMAI conference included in this special issue are far from giving a complete picture of the mutual impact between numerical optimization and real world problems. Nevertheless, they span a diverse range of topics and applications, and in particular they show that even mathematical issues, that at first glance appear to be very theoretical, can have application-related aspects.

This is the case for instance, of the generalization of the Féjer sequences in a real Hilbert space considered in [5]. The main novelty with respect to the standard

\section{Valeria Ruggiero and Gerardo Toraldo are the Members of INdAM Research group GNCS, Italy..}

Valeria Ruggiero

valeria.ruggiero@unife.it

Gerardo Toraldo

toraldo@unina.it

1 Department of Mathematics and Computer Science, University of Ferrara, via Saragat 1, 44122 Ferrara, Italy

2 Department of Mathematics and Applications R. Caccioppoli, Università degli Studi di Napoli Federico II, via Cintia, 80126 Napoli, Italy 
definition is the introduction of an additional term involving function values. The theoretical results obtained for this relaxed notion of Féjer monotonicity can be applied to analyze the convergence of several convex optimization methods for data mining in presence of an additional error term, due to the introduction of a variable metric or to stochastic perturbations.

In [1], using the theory about the functions satisfying the Kurdyka Lojasiewicz assumption, a block coordinate approach for minimizing the sum of a differentiable, not necessarily convex, function and non-smooth block separable terms is addressed. This problem arises, for example, in fluorescence microscopy, in emission tomography and in optical astronomy, where we have to perform blind deconvolution of images corrupted by Poisson noise and both the point spread function and the image have to be recovered. The method does not require the Lipschitz continuity of the gradient of the first term on the whole space and, combined with the Armijo search, enables to obtain global convergence results also in presence of inexact proximal inner steps.

An original contribution [4] is the use of the Infinity Computer Paradigm (ICP), in which a recently described infinite number called grossone is added to the real number system to enable the execution of computations involving either infinite or infinitesimally small numbers. The ICP is used to address the breakdown difficulties arising in Conjugate Gradient methods when dealing with possibly degenerate or indefinite linear systems. Indeed, it is proved that the analysis based on the geometric point of view of planar methods perfectly matches with the algebraic approach using the ICP.

Some applications motivate the development of novel algorithms and their implementations. In particular the design of complex engineering systems, such as a gas distribution network model, requires specially tailored methods for the solution of convex constrained nonlinear systems; for example, see the quasi-Newton algorithm developed in [8]. Similarly in [2], the problem of optimizing a ship's hull leads to a nonlinear constrained multi-objective optimization problem and the development of a hybrid multi-objective deterministic algorithm equipped with an efficient derivativefree local scheme.

In [6] a case-study of sparse regularization of a 3D CT reconstruction problem with limited/sparse data is proposed. In particular, the paper focuses on obtaining sufficiently good reconstructions in a very short time, well suited to clinical requirements, using a scaled gradient projection method equipped with very effective acceleration techniques.

The structure and the large scale of training problems for biased Support Vector Machines (SVM) motivate the development in [7] of a parallel decomposition method for box constrained quadratic programming problems with a single equality constraint. Exploiting the special form of the KKT conditions, the basic idea is to decompose the set of variables into disjoint blocks and to reduce the solution to a sequence of steps with the descent direction defined by the partial minimization of the cost function with respect to these blocks of variables. The auxiliary relatively small problems can then be solved in parallel.

We sincerely thank all the authors who submitted their papers, and the referees for their effort and very valuable contribution to this Special Issue. We also want to thank Prof. William W. Hager, Editor-in-Chief of Computational Optimization and 
Application for his support. Finally, we dedicate this Special Issue to the memory of our friend and colleague Roger Fletcher, who passed away in July 2016. Roger provided a unique and extraordinary contribution to the development of numerical methods which underlie many of the algorithms used for the solution of real-world problems.

\section{References}

1. Bonettini, S., Prato, M., Rebegoldi, S.: A block coordinate variable metric linesearch based proximal gradient method. Comput. Optim. Appl. (2018). https://doi.org/10.1007/s10589-018-0011-5

2. Campana, E.F., Diez, M., Liuzzi, G., Lucidi, S., Pellegrini, R., Picciarelli, V., Rinaldi, F., Serani, A.: A multi-objective DIRECT algorithm for ship hull optimization. Comput. Optim. Appl. (2017). https:// doi.org/10.1007/s10589-017-9955-0

3. Chandra, S., Jayadeva, Aparna, M.: Numerical Optimization with Applications. Alpha Sci. Int. Ltd. (2009). https://trove.nla.gov.au/work/28708667?q\&versionId=45319966

4. De Leone, R., Fasano, G., Sergeyev, Y.D.: Planar methods and grossone for the conjugate gradient breakdown in nonlinear programming. Comput. Optim. Appl. (2017). https://doi.org/10.1007/s10589017-9957-y

5. Liny, J., Rosasco, L., Villa, S., Zhou, D.-X.: Modified Féjer sequences and applications. Comput. Optim. Appl. (2017). https://doi.org/10.1007/s10589-017-9962-1

6. Loli Piccolomini, E., Coli, V.L., Morotti, E., Zanni, L.: Reconstruction of 3D X-rays CT images from reduced sampling by a scaled gradient projection algorithm. Comput. Optim. Appl. (2017). https://doi. org/10.1007/s10589-017-9961-2

7. Manno, A., Palagi, L., Sagratella, S.: Parallel decomposition methods for linearly constrained problems subject to simple bound with application to the SVMs training. Comput. Optim. Appl. (2018). https:// doi.org/10.1007/s10589-018-9987-0

8. Marini, L., Morini, B., Porcelli, M.: Quasi-Newton methods for constrained nonlinear systems: complexity analysis and applications. Comput. Optim. Appl. (2018). https://doi.org/10.1007/s10589-0189980-7

9. Yang, X.S., Koziel, S.: Computational Optimization and Applications in Engineering and Industry, Studies in Computational Intelligence. Springer, Berlin (2011). https://doi.org/10.1007/978-3-642-209864 